Sociologie et sociétés

\title{
Le politique du minoritaire
}

Étude de postures critiques d'un apparaître particulier à

travers les figures idéaltypiques du Juif et du Noir

\section{Politics of Minority}

\author{
A Study of the Critical Postures of a Distinctive Appearance \\ through the Ideal Types of the Jew and the Black
}

\section{Anaïs Sékiné}

Volume 42, numéro 1, printemps 2010

Les passeurs de frontières

Border Crossers

URI : https://id.erudit.org/iderudit/043966ar

DOI : https://doi.org/10.7202/043966ar

Aller au sommaire du numéro

\section{Éditeur(s)}

Les Presses de l'Université de Montréal

\section{ISSN}

0038-030X (imprimé)

1492-1375 (numérique)

Découvrir la revue

\section{Citer cet article}

Sékiné, A. (2010). Le politique du minoritaire : étude de postures critiques d'un apparaître particulier à travers les figures idéaltypiques du Juif et du Noir. Sociologie et sociétés, 42(1), 271-295. https://doi.org/10.7202/043966ar

\section{Résumé de l'article}

Cet article propose de mener une réflexion sur la tension dialectique minoritaire/majoritaire dans une analyse théorique, littéraire et microsociale de l'« apparaître » du minoritaire - l'apparaître étant le « qui » dans le « qui suis-je » qui se déclare ou se signifie dans l'espace social et public. Il s'agira d'appréhender les figures du Juif et du Noir comme des idéaltypes du minoritaire afin d'élaborer une dimension politique spécifique du minoritaire, qui se compose au sein d'une citoyenneté républicaine, individuelle, égalitaire et anonyme. Tout d'abord, nous nous efforcerons d'inscrire cette étude dans une perspective de sociologie politique et morale. Puis, afin de percevoir et d'analyser comment se discute et s'élabore le fait politique et ce qu'implique l'apparition du minoritaire dans le champ social, nous étudierons des textes choisis parmi certains dialogues, correspondances et essais dans le champ des études postcoloniales (Fanon, Hall) et de la " question juive " (Arendt, Sartre). 


\title{
Le politique du minoritaire
}

\author{
Étude de postures critiques d'un apparaître particulier \\ à travers les figures idéaltypiques du Juif et du Noir
}

\author{
ANAÏS SÉ KINÉ \\ Département de sociologie \\ Université de Montréal \\ C.P. 6128 Succursale Centre-ville \\ Montréal (QC) $\mathrm{H}_{3} \mathrm{C}_{3} / 7$ \\ Courriel: anais.sekine@umontreal.ca
}

\section{LES PASSEURS DE FRONTIÈRES}

La figure du passeur de frontières fait immédiatement penser à celle de l'étranger traitée par Georg Simmel dans sa fameuse «Digression sur l'étranger» (1984 [1908]). Pour Simmel, il est le commerçant, par lequel circule un réseau d'échanges de biens, monétaires, culturels. Il est le psychologue, en posture d'extériorité, à la fois proche et distant. Il présente l'avantage d'un rapport d'objectivité avec le groupe. À travers lui, le lointain se fait proche et le proche se fait lointain. Il est à la fois à l'intérieur et à l'extérieur. Il joue le rôle de médiateur. Il établit le passage entre l'autre et le soi, à l'entre-deux. Jamais complètement "des nôtres», il est celui qui arrive et celui qui reste et rappelle ainsi le groupe à son identité et à ses frontières. Il est représenté par la figure archétypale du Juif ${ }^{1}$, l'étranger par excellence dont le sociologue de l'École de Chicago, Louis Wirth (2006 [1928]), dresse le parcours sociohistorique à travers le ghetto. Si pour l'écologie urbaine, le ghetto est un espace de mobilité et de transition, il est aussi et surtout un espace de

1. Dans ce document, Juif et Noir prendront une majuscule. Le choix n'est pas totalement arbitraire. Il n'est pas question de stipuler l'existence d'un peuple ou d'une nation juive ou noire. Les concepts utilisés se prêtent simplement à ce choix délibéré mais pleinement discutable. 
séparation. À cet endroit, l'étranger ne gêne pas, car il n'est pas engagé dans la vie de l'établi, terme plus eliasien, celui qui est déjà là. Il n'est pas un égal.

Le passeur de frontière est aussi un homme marginal, figure développée par un autre sociologue de l'École de Chicago, et théoricien de la ville, Robert E. Park. L'homme marginal est le symbole, l'emblème de l'homme moderne. Cosmopolite, «citoyen du monde», il résout la synthèse des cultures et il est à l'avant-garde des sociétés modernes. Alors que l'étranger reste une figure en suspens, détachée du réel, l'homme marginal est pleinement inscrit dans l'espace commun, mais pris entre deux mondes. Il est de ces peuples «diasporiques» que conceptualisent plus récemment les études postcoloniales. L'homme marginal serait, selon Stuart Hall citant C.L.R. James, de «ces peuples qui sont dans la civilisation occidentale, qui y ont grandi, mais à qui l'on a fait sentir et qui ont eux-mêmes senti qu'ils étaient en dehors, ont un aperçu unique sur la société» (Hall, 2007: 264). L'homme marginal est l'un des «nôtres», mais pas tout à fait. C'est ce «pas tout à fait» qui appelle à la posture critique et à l'interpellation.

\section{PASSEURS RÉVÉLATEURS}

Dans ces deux figures de passeur — l'étranger et l'homme marginal — dans les spécificités de leur conceptualisation, il y a cette idée de différence. La différence n'existe pas en soi, elle ne peut être que dans la rencontre. Mais la légitimation du sens est dépendante de la marge de manœuvre dont le sujet dispose pour l'imposer, et ce, par jeu de domination. L'identité culturelle, dirait le théoricien du postcolonial Homi Bhabha, n'est pas question d'authenticité, mais d'autorité. Qui a l'autorité de cette identité (Bhabha, 1994)?

Ces figures révèlent tout à la fois un obstacle, une assignation, une limitation et une fluidité, un mouvement, une recréation, une présence et une absence, une visibilité et une invisibilité, une humanité universelle et un métissage d’humanité. "Métissage d'humanité», quel mot abject! L'expérience n'est jamais aussi violemment vécue que par ce trouble dans la définition de l'universel. C'est précisément la question qui nous intéresse ici, celle du trouble suscité par une telle apparition, celle d'un «dehors en dedans», de l'intrus dans la bergerie, du retournement de l'autorité. Et cet intrus, je l'appellerai le minoritaire. Dans le registre sociétal, le minoritaire est remarquablement inscrit dans l'espace commun. Le concept même de minoritaire ne se conçoit pas sans le majoritaire. Ils sont, et fonctionnent, en forme dialectique. L'un n'existe pas sans l'autre.

Le propos de cet article est de montrer comment cette apparition déclarative, cette révélation d'une frontière entre les hommes et leurs identités, est source d'une existence politique. L'existence, au sens étymologique d'ex-istensia en latin, «être placé» «hors de», au devant de soi-même, d'être dans le monde. Et une existence politique qui se crée par la rupture - car toute existence ne se fait pas politique - et une rupture située par une situation particulière, d'anormalité, d'oppression, qui est source d'un espace ouvert à un politique du minoritaire. En commençant par situer le propos politique, son ouverture à un «espace-qui-est-entre-les-hommes», et par expliciter les conditions et les implications de la situation minoritaire avec la sociologue (et féministe) Colette 
Guillaumin, on s'intéressera à des exemples de mises en scène subjectives et biographiques de la déclaration identitaire à partir de deux figures idéaltypiques du minoritaire: le Juif et le Noir. Le choix de ces «figures», interprétées à travers des témoignages littéraires, est motivé par le contraste de la visibilité. La visibilité, ou la non-visibilité, est un caractère différentiel déterminant, et soulève une problématique conceptuelle dont il est important de se saisir.

Commençons par la fameuse «question juive» sartrienne. Deux formes de discussion entre des philosophes du siècle dernier, à l'intérieur de deux contextes historiques distincts, offrent une illustration remarquable de la dialectique minoritaire-majoritaire. On s'intéressera, dans un premier temps, aux dialogues publiés par Jean-Paul Sartre et Benny Lévy, dans le retournement existentialiste de Sartre qui vaudra à Benny Lévy, dans les années 1970 en France, d'être accusé de détournement de vieillard. Puis on se penchera sur la correspondance tenue entre Hannah Arendt et Karl Jaspers, deux philosophes allemands pris dans la tourmente de la Seconde Guerre mondiale, dans des lettres échangées entre 1930 et $1952^{2}$. Dans un second temps, on élargira le propos à une autre problématique d'existence que Frantz Fanon opposera luimême à Sartre dans Peau noire, masques blancs (1952). Il oppose au Juif une visibilité radicale du Noir. Cette visibilité totale est ce qui l'empêche d'exister. Il est l'Homme invisible de Ralph Ellison (2002 [1952]). On abordera à partir de là les études postcoloniales et les travaux de Stuart Hall pour parvenir à problématiser ce couple contradictoire du minoritaire et du politique.

\section{CADRE CONCEPTUEL}

\section{1) Le point de vue minoritaire: une existence politique?}

La question veut se situer dans l'énoncé du fait minoritaire, dans celui qui apparaît dans l'espace majoritaire, non pas dans sa visibilité sensorielle immédiate, mais dans la dimension politique de celui qui parle «au nom de» son caractère minoritaire.

Qu'y a-t-il de politique dans une voix qui porte un nom, qui n'est pas libre, selon la définition de la cité grecque antique? Le politique est réservé à l'homme dans son unicité et sa voix propre et singulière. Selon Hannah Arendt, il «prend naissance dans l'espacequi-est-entre-les-hommes, donc quelque chose de fondamentalement extérieur-àl'homme» (Arendt, 1993: 42). Le politique du minoritaire serait le politique de l'esclave, de celui qui cherche à s'émanciper. L'émancipation est-elle un acte politique ou tout au plus, un devenir majeur, dans le sens kantien du minoritaire, celui qui est sous tutelle, en relation de dépendance? Aujourd'hui, poursuit Arendt, «nous sommes enclins à croire que la liberté commence où la politique finit» (Arendt, 1954: 193).

Le politique n'est pas une question de morale, civilisée, dogmatique, réglée, qui dit ce qui doit être. Le politique a une visée universelle à partir de son lieu, de son temps, de son monde socio-historique, à partir de ce qui fait communauté. Il ne peut

2. Les correspondances publiées de Hannah Arendt et Karl Jaspers sont comprises dans une période plus étendue allant de 1926 à 1969 (Arendt et Jaspers, 1985). 
donc être fixé, professé ni organisé. Le politique est en rapport à la Loi, au nomos. Celle qui nous gouverne et qui, pour autant, ne s'édicte pas et rappelle, quand elle est mise à mal, à l'exigence du jugement et de la responsabilité individuelle. Le nomos, pour les Grecs, c'est la norme universelle incontestable qui permet de faire de l'homme, un être humain. Le jugement, c'est la capacité anthropologique de l'homme à penser le bien et le mal, dans la pluralité de leurs sens et de leurs situations. Cette exigence est une problématique centrale pour tous ceux qui ont tenté de comprendre l'expérience du totalitarisme qui a saisi le $\mathrm{xx}^{\mathrm{e}}$ siècle. Dans son article et analyse du problème du «mal» chez Arendt, Dario De Facendis explique:

Le totalitarisme, [c'est] la capacité politique d'organiser ces masses dévaluées dans des mouvements politiques nés de la destruction même de l'espace politique. Le pouvoir qui en résulte est celui, absolu, de la non-valeur. (De Facendis, 2003: 87)

Quand la pluralité du monde humain est remise en cause, quand au sein du politique il n'y a plus d'espace pour un véritable débat sur le bien et le mal, mais qu'au contraire, bien et mal sont pensés comme étant nécessairement donnés par une quelconque construction logique du monde qui n'admet pas de variantes, alors, nous sommes témoins d'un processus de perdition de la qualité humaine des hommes. (De Facendis, 2003: 71)

Le politique exige une disponibilité à l'événement, à la nouveauté, à l'inconnu, au hors cadre, au non habituel. On peut y faire correspondre le concept lévinassien d' «action éthique», "qui s'oppose au geste de la répétition, souligne que l'homme est un nouveau commencement, un initiateur» (Ouaknin, 1992: 136). Ce concept de commencement comme natalité est un des concepts piliers de la théorie politique d'Arendt pour laquelle chaque naissance est un commencement, une nouvelle possibilité d'action et donc de liberté, d'extra-ordinaire et de nouveauté. Elle utilise même le terme de «miracle», «c'est-à-dire quelque chose à quoi on ne pouvait pas s'attendre (Arendt, 1958b: 220).

Parler «au nom de» est devenu la condition nécessaire et préalable à toute possibilité d'existence après les totalitarismes du $\mathrm{xx}^{\mathrm{e}}$ siècle qui ont tenté de les éradiquer. On s'interrogera sur la parole spécifique du minoritaire, son caractère inattendu dans le sens commun, dans le sens qui lie les hommes entre eux, au passage du connu et de l'imprévu, du semblable et de l'étranger. Le roman de l'auteur de littérature noire Richard Wright, Black Boy (1945) raconte, à partir du regard d'un jeune garçon noir du sud des États-Unis auquel il donne son propre nom, comment celui-ci décrypte son monde social et parvient à se considérer lui-même comme un représentant du genre humain, malgré la ségrégation et les humiliations. C'est son regard qui révèle la couleur de l'Amérique. Il en a fallu peu pour qu'il soit fiché au FBI en raison de son adhésion au Parti communiste en tant qu' «élément subversif» (Schofield, 2003). La littérature a été pour lui le moyen de transcender son apparaître immédiat, sa visibilité radicale, et d'intervenir dans le champ de la signification. La signification sans image de l'écriture a été en effet sa meilleure arme de subversion:

C'est sans doute pour cela que les sociétés dont la préoccupation première est de se définir comme strictement majoritaires portent leurs efforts sur la limitation de la liberté de 
l'écriture et de la publication, comme sur l'expurgation de l'enseignement. Le mythe de la société monolithique est la condition sine qua non de leur existence aussi bien que le fondement de leur idéologie. Boas, Mann, Einstein, brûlés par le nazisme, la Lorelei devenue anonyme, Euripide et Sophocle interdits par les colonels grecs, ou le cinéma américain par le régime de Vichy, en sont témoins. (Guillaumin, 2002: 184 en note)

En se nommant, le minoritaire se déclare. Il provoque un dérangement, un agacement. Il questionne l'ordre du monde et le bouleverse. Le minoritaire s'affirme, se revendique. Il entre dans la sphère publique, politique, et soumet des demandes spéciales, des «accommodements », dira-t-on au Québec. Il cherche à modifier les règles, à les conformer à soi, à un soi particulier et non général, dira le majoritaire, éloigné du bien de «tous». Le minoritaire s'affiche avec un problème. Il est collectif et exclusif. Il porte un projet qui concerne son existence propre. Or le minoritaire veut s'émanciper, devenir un homme universel, l'homme dont le projet est fondu dans l'individu. Mais en voulant le devenir, il en change le portrait et le récit. Son action identitaire se fait action politique. Qu'est-ce que provoque ce soudain apparaître?

\section{2) Dialectique minoritaire-majoritaire}

\section{Le minoritaire est d'abord une situation dans un ordre donné}

Dans cet article, le passeur de frontières est donc le minoritaire. Colette Guillaumin est sans doute celle qui a le mieux théorisé la situation minoritaire dans ce mécanisme idéologique. Dans L'idéologie raciste (2002), elle fait une analyse de texte, au mot à mot, des articles du quotidien France Soir et montre comment s'établit la «construction logique du monde» entre ordre anonyme dominant et excès de dénomination sémantique. Le minoritaire apparaît en effet dans le discours. Il n'apparaît qu'à travers lui. Il est «différent». C'est pourquoi il doit être nommé. Le minoritaire est celui qui porte un nom. Le majoritaire ne se nomme pas. Il est. Le «comme tout le monde» (collectif indéfini), «comme les autres» (pluriel indéfini), c'est le point de vue minoritaire. Du point de vue majoritaire, il n'y a pas de notion de totalité. Il est pure individualité. D'ailleurs pour lui, «tout le monde» n'a pas de sens. Il n'y a que des individus, certains qu'il décrit pourtant en les nommant (avec plus ou moins de censure) et d'autres qu'il qualifie par des particularités individuelles. L'idéologie raciste, qu'analyse Guillaumin, se situe précisément dans cette impasse sémantique.

Les minoritaires sont: "des groupes qui sont sociologiquement en situation de dépendance ou d'infériorité» (Guillaumin, 2002: 94-95). La situation minoritaire, c'est dans le même temps: «l'abstraction, c'est-à-dire le statut symbolique de sa minorité, et la réalité matérielle (la situation d'oppression)»(Guillaumin, 2002:122). On pourrait l'étendre plus largement à une situation de limitation des droits par rapport à la norme majoritaire, c'est-à-dire une limitation du potentiel de liberté établi par des normes culturelles, donc arbitraires (ex. : le droit au mariage homosexuel). Ce qui fait dialectique, c'est l'univers symbolique commun. Le minoritaire est défini dans l'espace majoritaire, donc dominant. Il n'est pas nécessairement défini par lui, mais sa définition 
dépend du statut du majoritaire. Plus encore, Guillaumin ajoute et insiste sur ce fait, «ses efforts pour se définir un système [propre] sont orientés et canalisés par le majoritaire; il ne peut se définir sur des références internes et indépendantes, il doit le faire à partir des références que lui offre le système majoritaire» (Guillaumin, 2002: 125). Le minoritaire est un individu qui n'a pas le droit aux mêmes droits et aux mêmes chances que les autres. C'est pourquoi aussi la reconnaissance de la diversité ne satisfait pas les politiques de droit à la différence. La différence a des conséquences. C'est une tactique démagogique qui n'aboutit à aucune amélioration sociale. Toutefois, la situation d'oppression dont parle Guillaumin est loin de se limiter à des considérations matérielles, bien qu'elles soient, dit-elle, «insécables de l'ensemble constitué par le symbolique et le matériel» (Guillaumin, 2002: 127). Le système de relations entre majoritaire et minoritaire est un système total, basé sur des situations de pouvoir inégalitaires. Le pouvoir, défini par Foucault, «ce n'est pas une institution, et ce n'est pas une structure, ce n'est pas une certaine puissance dont certains seraient dotés: c'est le nom qu'on prête à une situation stratégique complexe dans une société donnée» (Foucault, 1976: 123). La diversité n'est pas composée d'une juxtaposition de cultures, mais d'un réseau de relations antagoniques. Or, faut-il le rappeler, aucun élément, qu'il soit en situation de pouvoir ou en situation de domination, n'est lisible en dehors de ce système. L'aliénation idéologique n'est pas unidirectionnelle. Le pouvoir n'est pas la liberté. C'est ce qui fonde la dialectique minorité-majorité.

\section{Minoritaire et majoritaire sont compris dans un univers sémantique}

Les études d'histoire et de sociologie de l'immigration montrent aussi à quel point les statuts symboliques se modifient. Les cas "problématiques» changent de nom, changent de visage. Dans le schème marxiste où se situe Guillaumin, elle rappelle que les catégories sont le produit des relations sociales de production. Du mépris des paysans du XVII ${ }^{\mathrm{e}}$ siècle, on passe ensuite aux ouvriers à l'âge industriel, puis aux «Arabes» des colonies. Chacun a eu sa part de théorisation essentialiste, réduisant leur être productif à une nature. L'étude de Norbert Elias et John L. Scotson (1965) sur les Logiques d'exclusion démontre singulièrement le peu de crédit que constitue le «caractère» ou «attribut» naturel. Alors qu'aucune différence sociologique ne permet de distinguer un groupe de l'autre, le simple fait d'être arrivé après — dans un lieu de travail et de résidence - peut produire l'installation d'une logique hiérarchique et d'opposition entre établis et outsiders. La différenciation a dans ce cas conduit à des conséquences sociopsychiques de discrimination et de stigmatisation.

Or les catégories racisées sont-elles à ce point interchangeables? Dans sa propre élaboration conceptuelle de la situation minoritaire, le sociologue Pierre-Jean Simon (2006) remarque à raison que toute situation n'est pas équivalente et que dans une telle logique, on pourrait rapidement arriver à un phénomène inverse d'avoir du minoritaire partout et du minoritaire nulle part. C'est la fameuse formule tautologique du tout est dans tout et inversement. Il y a, de façon exemplaire dans mes deux propositions, juive et noire, une expérience historique mortifere: danger de mise à mort; esclavage, exécution, 
persécution, et extermination effective. Au contraire, il y a des spécificités qu'il est nécessaire de distinguer et de reconnaître dans la détresse concrète qu'elles impliquent comme dans leur singularité conceptuelle. Car le minoritaire est-il nécessairement défini par sa condition malheureuse? En effet, l'urgence que suscite le constat des discriminations délimite souvent le champ de la réflexion. L'analyse de Guillaumin est bien plus subtile, car elle ne s'arrête pas aux phénomènes concrets du racisme. Le point le plus probant de son épistémologie de la dialectique minorité-majorité se situe dans le champ sémantique. Car c'est là, à mon sens, que commence l'expérience minoritaire, comme majoritaire. Ce qui définit le minoritaire, c'est le caractère particulier. Caractère qui nomme l'altérité, l'univers du sens, le champ de la signification, compris dans l'imaginaire propre à une langue déterminée, «douée d'un sens qui est celui des implications propres à une société» (Guillaumin, 2002: 128).

\section{Le majoritaire est identifiable}

Le majoritaire se considère comme un Homme universel qui fait partie de la «généralité humaine» (Guillaumin, 2002: 165). Il est indéterminé et ne porte aucune autre particularité que sa singularité propre, son «caractère» disait Sartre. Au contraire, la particularité n'est pas «porteuse de la totalité humaine». Le Juif, la femme, l'homosexuel, le Noir, comme le singulier vient le signifier, sont totalement et imperturbablement juif, femme, homosexuel, noir.

L'apparition du minoritaire dans le champ de la signification, c'est aussi la possibilité de signifier «en creux» l'être majoritaire. Le majoritaire apparaît toujours dans la négativité, ce qu'il n'est pas. La subversion de Wright a été de révéler une dimension dérangeante du système majoritaire. En isolant les caractères qui ne nécessitent pas d'être mentionnés (par défaut), on peut rapidement arriver à un portrait assez précis du majoritaire: homme (pas femme), adulte (ni jeune, ni vieux), blanc (pas de couleur), athée/chrétien (pas religieux, pas juif, pas musulman), hétérosexuel (pas homosexuel), national (pas étranger), saint d'esprit (pas malade, ni fou, ni handicapé), de classe bourgeoise (pas pauvre) (Guillaumin, 2002: 295). Pour ne citer qu'un exemple, l'enquête du sociologue Éric Maurin sur Le ghetto français (2004) met bien en évidence que la ghettoïsation n'est pas à sens unique et que les contraintes sociales qui limitent l'accès à certains quartiers locatifs sont une forme de ghettoïsation silencieuse visant positivement à tenir éloignés les indésirables. Les enquêtes de ce type, dont les noms de Pinçon et Pinçon-Charlot (2007) sont les plus représentatifs, se font de plus en plus nombreuses bien que réduites. Elles mettent en évidence l'ethnocentrisme des majoritaires et des dominants et leur propre aliénation. «Classeurs classés par leurs classements», les distinctions opérées par les dominants déterminent tout autant leurs goûts et leurs opinions que ceux des dominés. La sociologie de Pierre Bourdieu ne serait-elle pas la meilleure illustration de la faible liberté (individuelle) des puissants — héritiers reproducteurs d'héritage? Guillaumin remarque d'ailleurs très justement que «l'ensemble des caractères de la minorité absolue ne se trouve constitué en groupe réel nulle part, et ne se découvre jamais que dans des individus particuliers, alors que l'ensemble des 
caractères de majorité absolue se trouve réuni dans un groupe réel» (Guillaumin, 2002: 123). Chacun, à sa place, peut être le minoritaire d'un autre, mais il arrive rarement, ou dans des cas isolés, que tous les caractères minoritaires soient concentrés en un groupe d'individus. Le minoritaire est variant. Or, bien qu'il n'existe pas d'unité majoritaire, il existe un groupe réel pour lequel la question minoritaire ne se pose pas. Le majoritaire ainsi figuré est totalisé. Le majoritaire est ordonné et porte à lui seul les valeurs de la société qu'il reproduit. Ce sont généralement des individus de ce groupe qui occupent les plus hautes fonctions du pouvoir. C'est généralement à eux aussi qu'incombe la responsabilité de l'oppression et son aveuglement. Un État qui met en place un ministère de l'Identité nationale est un État en crise qui ne veut plus prendre la charge de l'universel. La volonté de définir l'identité commune et de reconnaître leur prééminence, c'est aussi, paradoxalement, l'expression d'une difficulté à accepter la pluralité. Elle s'accompagne généralement d'un durcissement des modalités d'accueil de l'étranger.

\section{DU MÊME À L'AUTRE}

\section{1) Sartre et Benny Lévy: de l'existentialisme au messianisme}

\section{Contexte}

Il y a un caractère anormal à vouloir aujourd'hui se définir "quelque chose». Quand les Juifs accèdent officiellement à la citoyenneté française, après une succession de décrets en 1791 (à la même époque que les protestants et les anciens esclaves, mais bien avant les femmes), le judaïsme devait devenir une religion comme le christianisme, mais dénuée d'existence particulière. La judaïcité, le fait d'appartenir à une communauté juive, religieuse ou culturelle, était renvoyée au domaine privé, discret et indistinctif, en d'autres termes, rendue invisible et inconséquente dans le monde commun. La déclaration du comte de Clermont-Tonnerre symbolise clairement les conditions de l'égalité et de l'intégration.

Il faut refuser tout aux juifs comme nation et accorder tout aux juifs comme individus; il faut méconnaître leurs juges, ils ne doivent avoir que les nôtres; il faut refuser la protection légale au maintien des prétendues lois de leur corporation judaïque; il faut qu'ils ne fassent dans l'État ni un corps politique ni un ordre; il faut qu'ils soient individuellement citoyens. (Comte de Clermont-Tonnerre, $1789^{3}$ )

Cette condition d'accès à la citoyenneté, pour le moins contraignante, aurait dû logiquement faire disparaitre la visibilité des Juifs en tant que groupe au fur et à mesure de leur assimilation. Le minoritaire est voué à l'émancipation.

Cette conception de l'identité particulière est toujours vivace en France. Quelques anecdotes sur les pensées communes, et sans aucun doute générales, illustrent simplement ce fait. Un lecteur du blogue de Nicole Lapierre (2009) se révolte contre son billet sur la judéité de Lévi-Strauss: «Lévi-Strauss était juif? J’ai du mal à l'imaginer pratiquant à la synagogue.» Il ajoute ensuite: «La judéité n'existe tout simplement pas, pas

3. Comte Stanislas de Clermont-Tonnerre, cité dans Poliakov (1968: 234). 
plus qu'une très hypothétique francité.» Lors du débat qui a suivi la projection, en juin 2008, du dernier documentaire d'Isy Morgensztern au Musée d'Art Moderne et Contemporain de Strasbourg, «Benny Lévy. La révolution impossible (La loi du retour)» (Morgensztern, 2008), un intervenant dans la salle affirmait que le choix de Benny Lévy d'immigrer à Jérusalem signifie un strict retour au religieux. Il ne s'agirait que de la conversion d'un personnage politique révolutionnaire, Benny Lévy — alias Pierre Victor, le chef de la gauche prolétarienne - en un Juif orthodoxe. Dès lors, Pierre Victor n'est plus et Benny Lévy devient un Juif, limitatif, auquel un non-Juif ne peut plus se relier. "Religieux», Pierre Victor devient le Juif orthodoxe Benny Lévy, parti à Jérusalem et dont on ne parle plus. Les dialogues publiés par Sartre et Benny Lévy sont en ce sens éloquents.

\section{Le retournement sartrien}

À l'époque des Réflexions sur la question juive, en 1946, Sartre cherchait surtout à faire du Juif un homme commun, « un homme, pas un Juif», un Homme universel. La publication de son essai a été d'une grande importance en ce qu'elle brisait le silence de l'aprèsShoah et s'adressait aux non-Juifs pour démontrer la mythologie de l'antisémitisme.

Le Juif est un homme que les autres hommes tiennent pour Juif: voilà la vérité simple d'où il faut partir. (Sartre, 1946: 83-84)

Benny Lévy détaille la proposition de Sartre. Le Juif est surdéterminé, car la judéité s'ajoute à l'altérité qui distingue chaque individu: «le Juif a comme nous un caractère et par-dessus le marché, il est Juif» (Sartre, 1946: 96). Le Juif est immobilisé, car il ne peut se défaire de sa condition. Il est fatalement Juif et confronté à la solution de se reconnaître et d' «être responsable dans et par sa propre personne du destin et de la nature même du peuple juif» (Sartre, 1946: 108), ou de se nier «désespérément». Le Juif est, simultanément, celui qui, à la question «qui es-tu? », répond: «Je suis Juif», et celui qui ne peut pas répondre «Je ne suis pas Juif». Sartre forme ainsi les deux êtres juifs: le Juif authentique qui assume son identité et le Juif inauthentique qui passera sa vie à la contester ${ }^{4}$.

À cela Levinas proteste, rapporte Benny Lévy: «Être juif pour être juif, cela ne vaut pas la peine ${ }^{5} »$ (Lévy, 2003: 36). Le politique se réduirait alors à de la réactivité, à une assignation subie ou à une passivité mortifère, vidée de toute possibilité d'action (donc a-politique). Pour Levinas, et d'une différente manière pour Arendt (1951: 18-19), l'être juif et l'historiographie juive sont porteurs d'un projet ${ }^{6}$.

4. Bien qu'Arendt ait exprimé une grande hostilité vis-à-vis de Sartre, ses concepts de paria conscient et de parvenu ne sont pas très éloignés de la typologie élaborée par Sartre.

5. Levinas ne répond pas ici à Sartre, mais à Jérôme Lindon et à Emmanuel Berl qui arrivent à la même conclusion et avec lesquels il dialogue.

6. En réponse à Sartre, Arendt parle de «conscience juive» indépendante « des autres hommes » qui définiraient le Juif selon Sartre. À propos de l'idée de projet, voir aussi l'historiographie juive de Yéroushalmi (1982). 
C'est ainsi que Sartre revient sur ses propos dans un entretien avec Benny Lévy qu'ils publient sous le titre: L'espoir maintenant (Sartre et Lévy, 1991) ${ }^{7}$. "À l'heure qu'il est, je pense qu'il y a une réalité juive par-delà les ravages de l'antisémitisme sur les Juifs. (...) Le Juif se considère comme ayant un destin» (Sartre et Lévy, 1991: 67).

Le religieux est une relation métaphysique au destin du monde, le monde dans lequel nous vivons tous, Juifs, non-Juifs, minoritaires, majoritaires, Masaïs et Bretons. Il n'y a plus de séparation du politique et du religieux, car judaïsme, Christianisme et marxisme, que Sartre mentionne et compare, sont tous envisagés également d'un point de vue religieux, philosophique, politique et éthique. Il les envisage en dehors de leur cadre dogmatique. C'est une recherche d'un sens à l'histoire. C'est pourquoi il n'est pas utile d'entrer plus en détail, à ce niveau, dans l'explication religieuse, car il ne s'agit pas ici de donner une interprétation juive et judaïque de l'existence juive. Elle est une existence énoncée par le discours et active par les faits, et non par l'étude talmudique. L'explication sartrienne vaut pour elle-même matière d'analyse d'un point de vue extérieur. L'extériorité n'est pas seulement due au fait de sa non-judéité, mais bien plus fondamentalement parce que Sartre n'a jamais étudié le corpus hébraïque ${ }^{8}$.

\section{Impossible révolution: pensée politique de l'infini et de la naissance}

Benny Lévy intervient relativement peu dans la discussion. Il dirige l'entretien et fait parler Sartre pour qu'il détaille au plus près sa pensée. Sartre conçoit le Juif avec le judaïsme. L' «unité de la réalité juive» est, selon Sartre, un rapport particulier avec l'unité divine, une «liaison métaphysique avec l'infini» (Sartre et Lévy, 1991: 71). Il résume: «Toute l'histoire des juifs consiste justement en ce premier rapport.» «L'essentiel, c'est que le Juif a vécu et qu'il vit encore métaphysiquement» malgré l'apparition d'un deuxième monothéisme et malgré l'histoire. Ce qui l'interpelle dans le judaïsme, c'est sa finalité messianique. Elle est «l'apparition de l'existence éthique des Hommes les uns pour les autres» (Sartre et Lévy, 1991: 73), non plus comme responsabilité, mais comme évidence, par le dépassement des règles, précise Benny Lévy, et non par leur transgression. Benny Lévy déplace le débat de sa politique, il change le destin de sa révolution. Une révolution qui, si on le croit, aurait pu, en 1973, basculer dans une guerre terroriste. Une révolution, néanmoins, qu'il choisit de mener par la pensée. L'infini, pour Levinas, aussi étrange que cela puisse paraître dans nos représentations raisonnées et raisonnables, «ce n'est pas l'orgueil d'un nationalisme exacerbé par les persécutions. L'indépendance à l'égard de l'histoire affirme le droit que possède la conscience humaine de juger un monde mûr à tout moment pour le jugement, avant la fin de l'histoire et indépendamment de cette fin, c'est-à-dire un monde peuplé de personnes» (Levinas, 1963: 302). Entrer dans ce schème, c'est résoudre la dialectique majorité-minorité. Ce pour quoi, sans doute, Benny Lévy a choisi d’être un Juif parmi

7. La collaboration de Jean-Paul Sartre avec Benny Lévy a conduit à un retournement considérable de la pensée sartrienne de l'existentialisme, ce qui a été vivement remis en question et suscité une polémique.

8. Ce qu'il regrette, dit-il, mais qui demandait trop de temps (apprendre l'hébreu) au vu de son âge avancé et de ses problèmes de santé (il est quasiment aveugle au moment où il rencontre Benny Lévy). 
les Juifs, et non un Juif de la diaspora, un Juif parmi les non-Juifs. Ce messianisme est une pensée de la révolution et permet à Sartre de repenser la révolution marxiste par un dépassement des préoccupations économiques, pour une fin éthique, c'est-à-dire, la révélation de la pluralité ${ }^{9}$. L'être juif avec le judaïsme résout la contradiction du Juif imaginaire. Il a une existence.

Ce destin est lié à la naissance. Le « rêve de Sartre était d' «humaniser la naissance». (...) l'homme comme fils de l'homme» (Lévy, 2002: 290-291). Benny Lévy dira «Vous aurez beau devenir sociologue, révolutionnaire, Juif réformé, vous ne changerez rien à ce fait foncier, fondamental, initialement et destinalement: vous êtes nés, du début à la fin» (Lévy, 2003: 43). Naître, c'est naître situé dans un ordre du monde. On ne naît pas avec rien. Le rien n'existe pas, car il serait une négation de l'existence. L'indétermination de l'homme qui apparaît avec la démocratie n'est pas un recommencement perpétuel de la naissance du rien par le rien. Naître c'est venir au monde, exister avec un il y a.

L'être juif est factice, mais c'est cette contingence qui rend possible l'existence. L'irrémissibilité de l'être juif, c'est-à-dire son caractère impardonnable dans le totalitarisme, c'est l'irrémissibilité de l'être humain, précisément, la réalisation du néant, la dé-création. La poursuite de l'aryanisme ne va pas vers une détermination idéale de l'être humain, mais au contraire vers une indétermination absolue. D'où les affinités entre la forme totalitaire de la société et la forme démocratique, théorisées par Arendt, mais dont on peut voir déjà les fondements dans De la démocratie en Amérique d'Alexis de Tocqueville (1835 et 1840) qui identifie la contradiction démocratique (liberté/ égalité/pouvoir anonyme du peuple/omnipotence).

\section{2) Contexte de survie: dialogue entre Hannah Arendt et Karl Jaspers}

\section{Contexte}

Au début du $\mathrm{xx}^{\mathrm{e}}$ siècle, et depuis le décret d'émancipation des Juifs en 1791, l'entrée tardive des Juifs dans l'histoire des peuples nationaux maintient une frontière symbolique qui ne les assimile jamais tout à fait, quelle que soit l'ancienneté des familles juives et quel que soit leur degré d'acculturation ou de déjudaïsation. Arendt personnifie cette contradiction par la vie de Rahel Varnhagen, Juive allemande prise dans le paradoxe de l'assimilation du XIx ${ }^{\mathrm{e}}$ siècle (Arendt, 1958a). «Sortir du judaïsme» sonne comme une prescription pour tous les Juifs émancipés de ce siècle. Or, aucune tradition ne lui a été transmise. Privée de mémoire, elle est livrée à l'espace vide du paria qui n'est plus ce qui a été et ne s'inscrit dans aucune histoire. Rahel est pure individualité, «qui n'est que ce qu' [elle] a (Arendt, 1946: 48), son expérience: "À cette originalité [...] s'alimente chez Rahel [...] le vide de celui qui sans cesse doit s'en remettre aux expériences et qui a besoin de toute la vie pour forger chacune de ses opinions» (Arendt, 1946: 44). Sous plusieurs aspects, ce travail est, selon la biographe d'Arendt, Élisabeth

9. Sartre mêle régulièrement les termes moral et éthique comme s'ils étaient interchangeables. Leur distinction est certes un sujet en soi. Elle se situe peut-être dans le dogme que l'éthique ne comprend pas. La morale dicte ce qui est le bien, l'éthique est la responsabilité du bien. Elle ne dit pas ce qu'il est. 
Young-Bruehl (1982), un travail autobiographique. Hannah Arendt considère sa judéité en dehors du judaïsme, une judéité déjudaïsée. Comment envisager sa natalité dans ce cas autrement que par un fatalisme, une vacuité surdéterminée?

Une correspondance particulièrement éloquente quant au propos de cet article est celle tenue entre Hannah Arendt et Karl Jaspers de 1926 à 1969, c'est-à-dire de l'avant-guerre à l'après-guerre, donc avant et après la Shoah. Inutile de rappeler les «modalités» réservées aux étrangers et autres hommes marginaux à cette époque et en ces lieux. Bien que cette discussion se tienne entre deux individus dont la tradition (philosophique) allemande est incontestable, la position qu'occupe chacun d'eux Jaspers, défenseur d'un idéal allemand, et Arendt, Juive exilée dont seule la langue est porteuse de cet idéal - permet de reconstituer l'opposition qui peut exister entre un établi et une outsider dans d'autres contextes, furent-ils français. Les termes et les critères employés coïncident avec les débats qui ont lieu aujourd'hui. La question, et la problématique différence qu'elle instaure entre eux, a le mérite de mettre tout de suite en situation.

Jaspers pose la question de la différence entre un Juif allemand et un Allemand, là où pour lui, l'un et l'autre peuvent se confondre sans ambiguïté. Pour lui, un Juif allemand est allemand. Jaspers exprime ici la dynamique qui a été celle même des Juifs allemands au XIX ${ }^{\mathrm{e}}$ siècle, profondément dévoués à leur assimilation, au point de ne pouvoir admettre le péril du nazisme jusqu'à la limite de l'indéniable. Cette question est posée une première fois au début des années 1930 (Arendt quitte l'Allemagne en 1933) et la discussion reprend en 1947. Les dates de correspondance entre Arendt et Jaspers, comme celles de l'écriture des textes, essais théoriques et articles d'Arendt, sont importantes, car elles ne renseignent pas seulement sur une époque. Toute l'œuvre d'Arendt est une tentative de "penser l'événement». C'est pourquoi, peut-être, elle résistait à l'appellation "philosophe», car son intention n'était pas de fonder un système philosophique. Son problème philosophique était éminemment politique et donc mené par une conscience du présent et l'effort de comprendre son temps de manière globale. La question «qu'est-ce qu'être juif?» constituait une problématique centrale dans le contexte nazi, postnazi, de la reconstruction de l'Allemagne, de la création de l'État d'Israël en 1948 et avant cela, de tout ce qui concerne ladite émancipation des Juifs en Europe, depuis leur accession à la citoyenneté en 1791 en France, puis progressivement dans toute l'Europe occidentale.

En 1933, Jaspers défend une conception de l'Allemagne qui évoluera avec le temps et l'irrévocable de l'événement qui suivra. L’Allemagne est pour lui un idéal supranational dont il retrouve le caractère dans d'autres pays limitrophes qu'il intégrerait dans un «empire des Allemand ${ }^{10}$ ». Son idée du «caractère allemand» a une "visée totalisante, historiquement indéfinie». En d'autres termes, un peuple serait le garant d'un

10. Lettre de Karl Jaspers à Hannah Arendt, 3 janvier 1933, in Arendt et Jaspers (1985: 40-42). 
idéal universel porteur d'une mission en faveur «de la civilisation de l'avenir ${ }^{11}$ ». L'«essence allemande», sa grandeur, sa philosophie, sa littérature, son université, est ce qui devrait naturellement amener Arendt à dire, «c'est ainsi que je veux être allemande». C'est ainsi, selon lui, qu'elle est allemande, car elle en partage la tradition, la culture. Il lui suffit d'adopter, et de s'identifier au destin historique et politique de l'Allemagne. Or, s'étonne-t-il, «je suis surpris qu'en tant que Juive vous vouliez vous distinguer de ce qui est allemand $»^{12}$.

\section{2. «Une différence entre nous»}

En 1947, le discours de Jaspers change quelque peu de nature. De «l'essence allemande, il ne reste en effet que la langue ${ }^{13} »$, regrette-t-il. «Vous et moi avons pris conscience en 1932 (...) d'une différence entre nous, que je n'ai pas considérée comme personnelle à l'époque, une différence qui n'est pas absolue en soi, mais qui n'est pas pour autant une bagatelle ${ }^{14}$.» En quoi est-elle personnelle, il ne l'explique pas clairement. Il s'agit pour lui d'un certain ordre du monde. Bien que l'on puisse volontairement, par sa pleine conscience, choisir une nation, c'est-à-dire une responsabilité politique, un État, «il y a cependant quelque chose qu'on ne peut choisir, mais qu'il faut assumer ${ }^{15}$ ». Il est allemand, réfléchit constamment avec son cœur sur ce que signifie pour lui être allemand, non seulement par conviction, mais parce qu'en 1947, le monde le lui crie. C'est ainsi qu'il apparaît au monde, et tient à assumer la responsabilité de ce nom. Il ne se reconnaît pas en tant que coupable. Il n'est pas question de cela. Il s'agit pour lui de "culpabilité métaphysique» qui, selon Arendt, "recèle non seulement l' "absolu", où l'on ne peut effectivement plus reconnaître aucun juge terrestre, mais aussi, cette solidarité (qui selon la formule de Clémenceau: “L'affaire d'un seul est l'affaire de tous") constitue le fondement politique de la république ${ }^{16}{ }$. Il se confronte néanmoins à sa propre contradiction lorsqu'il exprime toute sa difficulté à définir ce qui est précisément de l'ordre de l'indéfini, de l'interprétation, de la représentation, mais délimite en retour ce qu'est un Juif, «c'est la religion biblique et l'idée de Dieu et l'idée d'Alliance, sinon, il me semble que le Juif cesse d'être juif ${ }^{17}$ ».

La condition sociale des Juifs en Allemagne n’était définitivement pas la même que celle des non-Juifs: «Je me souviens très bien de notre désaccord lorsque vous avez dit ou écrit un jour que nous étions tous dans le même bateau. [...] Avec Hitler pour capitaine, $[. .$.$] nous autres Juifs ne serions plus assis dans le même bateau.» Et ajoute-t-$ elle: «Ça aussi c’était faux, car dans ces conditions vous-mêmes n’étiez plus non plus

11. Lettre d'Hannah Arendt à Karl Jaspers, $1^{\text {er }}$ janvier 1933, (ibid.: 40), citation par Arendt d'une expression employée par Jaspers dans son livre Max Weber. Deutsches Wesen im politischen Denken in Forschen und Philosophieren, Oldenburg, 1932.

12. Lettre de Karl Jaspers à Hannah Arendt, 3 janvier 1933; ibid.: 40-41.

13. Lettre de Karl Jaspers à Hannah Arendt, 8 janvier 1947, ibid.: 54.

14. Lettre de Karl Jaspers à Hannah Arendt, 16 mai 1947, ibid.: 57.

15. Lettre de Karl Jaspers à Hannah Arendt, 20 juillet 1947, ibid.: 105.

16. Lettre d'Hannah Arendt à Karl Jaspers, 17 août 1946, ibid.: 51.

17. Lettre de Karl Jaspers à Hannah Arendt, 20 juillet 1947, ibid.: 106. 
dans le bateau — ou tout au plus comme dans une prison ${ }^{18}{ }^{»}$. Jaspers est en rupture, mais en rupture avec sa propre globalité qui est l'Allemagne. Arendt est dans un autre espace, l'espace d'entre-deux créé par sa condition. C'est une première différence que l'on peut aussi comprendre par la question de la responsabilité politique, au lendemain de la guerre qui a accéléré la création de l'État d'Israël en 1948 :

Si aujourd'hui les Juifs allemands ne veulent plus être Allemands, on ne peut sûrement pas nous en faire grief, mais, naturellement, cela a aussi quelque chose de comique. Ce qu'ils veulent dire en fait, c'est qu'ils n'envisagent pas de partager la responsabilité politique avec l'Allemagne; et en cela ils ont raison. Et c'est cela qui est décisif. Voyez-vous, il va de soi aujourd'hui, pour moi et pour beaucoup d'autres, que lorsque nous ouvrons le journal, nous vérifions d'abord ce qui se passe en Palestine — bien que je n'aie pas l'intention d'y aller $[\ldots]^{19}$.

Enzo Traverso, dans ses recherches sur l'histoire intellectuelle des Juifs entre le XIX ${ }^{\mathrm{e}}$ et le $\mathrm{xx}^{\mathrm{e}}$ siècle, rapporte que «l'ensemble du contexte social poussait objectivement l'intelligentsia juive à assumer une attitude anticonformiste, critique vis-à-vis de l'ordre établi» (Traverso, 1997: 57). Devant l'antisémitisme prévalant dans les universités et à l'impossibilité d'une carrière dans les institutions publiques, l'engagement «politique» de ces intellectuels passait notamment par une créativité artistique remarquable dans cette période, à Berlin, Vienne et Prague (Traverso, 1997: 60). Dans le milieu où Arendt évoluait, son entourage se divisait entre les affinités politiques sionistes ou communistes. Le mouvement ouvrier, militant pour la liberté, ne faisait aucune distinction entre les Juifs et les gentils. Il constituait la rencontre parfaite entre la lutte contre le racisme et la possibilité de s'y engager en tant qu'individu neutre. Et c'est dans cette alternative que s'est organisée la vie sociale d'Arendt (Young-Bruehl, 1982). Son mari, Gunther Stern, fréquentait les milieux communistes, son ami Walter Benjamin et son futur mari Heinrich Blücher défendaient les idées internationalistes. Ce fut surtout avec son ami Kurt Blumenfeld qu'elle s'engagea activement dans le mouvement sioniste. Il organisait « un sionisme destiné en premier lieu aux Juifs qui — à la différence des Juifs de l'Est - avaient connu dans leur histoire l'émancipation et l'assimilation et qui, du coup, ne pouvaient s'appuyer sur aucune existence communautaire, religieuse et sociale, pour lutter contre l'antisémitisme» (Young-Bruehl, 1982: 92). Ce fut justement lui «qui éveilla et entretint [l'] identité juive [d'Arendt] et qui lui fit partager le renouveau de la conscience juive, entrepris par les sionistes» (Young-Bruehl, 1982: 88).

Ainsi, sur le plan de l'identité, la définition ne peut être aussi limitative et théorique que dans l'ordre du religieux et de son allégorie, car, dit-elle, «le fait est néanmoins que beaucoup de Juifs sont comme moi totalement indépendants du judaïsme et sont pourtant des Juifs ${ }^{20} »$. Elle suggère alors que la judéité n'est pas seulement de l'ordre de la pratique, mais de l'existence, serait-elle politique. Le laconisme de la réponse

\footnotetext{
18. Lettre d'Hannah Arendt à Karl Jaspers, 30 juin 1947, ibid.: 103.

19. Ibid.: 104.

20. Lettre d'Hannah Arendt à Karl Jaspers, 4 septembre 1947, ibid.: 108.
} 
d'Arendt désempare ses lecteurs, tout comme ses exégètes Richard Bernstein (1996) ou Martine Leibovici (1998), qui questionnent ce que peut être une judéité sans judaïsme (et peut-on ajouter, un sionisme sans sionisme).

\section{Une judéité déjudaïsée}

Si le judaïsme religieux, par ses rites et ses coutumes, n’a jamais été central dans sa vie familiale, à la question: «Qui êtes-vous?» Arendt répond néanmoins «Une Juive», comme le postulat de sa mise au monde, à partir de quoi tout commence. Sa judéité relève pour elle d'une évidence, comme un fait quasi immanent. «Vous appartenez toujours à un groupe quelconque de par votre naissance», rétorque-t-elle à Günter Gauss, le 28 octobre 1964, lors d'un entretien télévisé (Arendt, 1946: 246). Or, ce postulat n'a aucun contenu prédéterminé. Il n'est question ni d'essence ni de fatalité. Qu'est-ce alors qu'être juive pour Arendt?

La première fois qu'Arendt a eu à répondre de cette appartenance, c'est quand, petite fille, on lui a fait remarquer qu' elle «n'avait pas l'air comme les autres». Il aurait été absurde pour elle de le nier. Or devant Jaspers, elle a bien l'air comme lui, elle est son égale. Et il lui faut revendiquer cette différence, au risque de disparaitre. Par son travail sur la vie de Rahel Varnhagen, elle veut «montrer que le fait d'être juif rend possible une certaine existence ${ }^{21} »$. Elle n'essaye pas de «fonder» l'existence de Rahel sur sa judéité, comme l'interprète tout d'abord Jaspers. En 1952, une fois le livre achevé, l'appréciation de Jaspers change en effet: «Cette figure, vous lui donnez la parole, mais pas à partir d'un centre, celui de l'être humain lui-même, qui n'est pas essentiellement juif, mais qui passe dans ce monde en tant que Juif et vit de ce fait le pire, lequel n'arrive pas seulement au Juif ${ }^{22}$.»

Jean-Luc Fidel, dans sa présentation à l'édition réduite de la correspondance, qualifie l'analyse de Jaspers de «professorale». En effet, le caractère professoral tient au fait qu'il tend à généraliser un «destin » individuel qu'il élève en «figure», ou, pourraiton ajouter, en «idéaltype» objectivé. Le «pire» n'est certainement pas l'attribut de l'expérience juive, mais la conclure ainsi revient à la banaliser par effet de comparaison, et vide l'histoire et le politique de toute signification. La reconnaissance de la spécificité est primordiale pour comprendre la problématique de l'exil par exemple, et au-delà, celle de la responsabilité et du jugement, deux notions fondamentales dans la pensée d'Arendt (2005), pour ne pas être seulement un autre parmi les autres. Toute appartenance, affiliation, identification ne s'équivaut pas. La radicalité par laquelle le totalitarisme a tenté d'évincer toute trace d'altérité — et pas seulement juive — rappelle néanmoins la nécessité de reconnaître l'autre dans son existence propre. Cette nécessité concentre toute la problématique du minoritaire et de sa politique.

21. Lettre d'Hannah Arendt à Karl Jaspers, 24 mars 1930, op. cit.: 102.

22. Lettre de Karl Jaspers à Hannah Arendt, 23 août 1952, op. cit.: 111-112. 
L'affirmation de sa judéité vient souligner le droit d'exister en tant que tel et de répondre de ce nom dans toute situation, qu'elle soit dans la condamnation du fonctionnaire nazi Eichmann, comme dans celle de la coopération des Conseils juifs (Arendt, 1963). D'une part, elle ne revient pas du procès où l'avait envoyé le New York Times avec la description du monstre que l'on attendait d'elle. Au contraire, ce procès lui a permis de se rendre compte de «la banalité du mal», de la normalité déstabilisante du haut fonctionnaire nazi Adolf Eichman, «un citoyen respectueux de la loi» (Arendt, 1963: 1149), «une personne moyenne, normale, ni faible d'esprit, ni endoctrinée, ni cynique [...] absolument incapable de distinguer le bien du mal» (Arendt, 1963: 1044). D'autre part, c'est elle qui est accusée de manque de compassion et de haine de soi quand elle écrit, «toute la vérité, c'est qu'il existait des organisations de la communauté juive, des associations de secours et d'entraide à l'échelle tant nationale qu'internationale. Partout où les Juifs vivaient, il y avait des dirigeants juifs, reconnus comme tels, et cette direction, presque sans exception, a coopéré, d'une façon ou d'une autre, pour une raison ou pour une autre, avec les nazis» (Arendt, 1963: 1139). Aucune extériorité ne permet de comprendre les raisons de ce choix de prendre part à la machine nazie. Pensant sans doute qu'ils pourraient mieux négocier la vie d'un certain nombre contre celle d'un certain autre, les Conseils juifs ont, selon Arendt, facilité le travail administratif et le rendement des déportations. En d'autres termes, en mettant de l'ordre dans le chaos, en jouant le jeu du dominant, le minoritaire s'est annihilé. La polémique qu'a suscitée la publication de son livre montre à quel point Arendt déstabilise le cadre des catégories de pensée. Son jugement est dur, peut-être, ou sans doute, injuste. Mais il engage une nécessité de conscience et de responsabilité pour ne pas faire de l'histoire un mythe.

La «question juive», ou le «nom juif» n'est pas la question des Juifs, mais c'est bien par la judéité qu'elle se pose. Pris individuellement, les Juifs, quelle que soit leur position sociale, privée ou publique, sont traversés vis-à-vis de leur judéité par l'interpellation de cette existence spécifique, collectivement nommée, qu'ils la rejettent ou qu'ils l'acceptent. Sociologiquement ou historiquement, les Juifs ne sont pas confrontés au choix de leur identité, mais plutôt à la responsabilité des actes, des représentations et des associations qu'ils sont prêts ou non à assumer sous ce nom selon leur propre jugement. Être juive, pour Arendt, est une responsabilité éclairée de son être né dans le monde qui est le sien, être totalement dans la liberté de ce qui lui appartient.

\section{LE SPECTACLE DE L'AUTRE}

À cette liberté qui incombe à Arendt, à la natalité qu'elle intègre pleinement dans sa vie, à la double révolution de Pierre-Benny Victor-Lévy, Fanon leur oppose une visibilité inaltérable, une impossible transformation, un ordre irrévocable. Sa différence à lui fait spectacle. Sa natalité est prédestinée. Partout depuis l'impérialisme européen, le Noir est soumis à son extrême contraste sensoriel. Le Noir est paralysé par l'extrême vacuité de son identification. La distinction porte à réflexion. 


\section{1) Réponse de Frantz Fanon à Jean-Paul Sartre}

\section{L'impossible rupture des damnés de la terre}

Fanon reprend la logique sartrienne des Réflexions à son compte. Pour le majoritaire sartrien, le racisé n'existe que parce qu'il y a un raciste. Autrement, il est un homme comme les autres. À cela, Fanon renvoie précisément le Juif à sa facticité, celui qui aurait pu ne pas être. À l'irrémissibilité de l'être juif, Fanon répond:

Le Juif peut être ignoré dans sa juiverie. Il n'est pas intégralement ce qu'il est. On espère, on attend. Ses actes, son comportement décident en dernier ressort. C'est un Blanc, et, hormis quelques traits assez discutables, il lui arrive de passer inaperçu. (Fanon, 1952: 93)

Le Juif est juif par hasard, et d'ailleurs, il peut vivre comme s'il ne l'était pas. D'autres n'ont-ils pas annoncé après lui que les Juifs sont en effet devenus des white folks, à l'instar des Irlandais aux États-Unis ${ }^{23}$ ? Fanon établit un écart dans l'effet de réalité entre une appréhension radicale de la visibilité et ce qui nécessite une reconnaissance plus médiate. Autrement dit, cette reconnaissance mobilise un savoir plus élaboré et donc moins efficace. L'insoumission aux critères de la race construite par le raciste est peut-être plus insupportable quand cet autre racisé ne répond pas à ses critères.

Un peu plus loin sur la même page, Fanon définit la différence avec le Noir:

Le Juif n'est pas aimé à partir du moment où il est dépisté. Mais avec moi, tout prend un visage nouveau. Aucune chance ne m'est permise. Je suis sur-déterminé de l'extérieur. Je ne suis pas l'esclave de «l'idée» que les autres ont de moi, mais de mon apparaître. (Fanon, 1952: 93)

Selon lui, sa détermination est totale et définitive. Noir, Martiniquais, psychiatre et écrivain, il s'adresse à un Blanc, Français de la métropole, philosophe de l'existentialisme. La Martinique est une culture créole, issue d'une rencontre inégalitaire entre colons blancs et colonisés (autochtones ou déplacés). Une culture diasporique tant l' "Afrique» y constitue une origine essentielle, originelle et immanente, portée par la trace de la couleur. Le noir comme stigmate de cette expérience originelle et traumatique. Fanon interpelle et rappelle la condition de l'être noir qui prend selon lui (au risque d'une extrapolation), un effet de réalité qui se passe de discours, d' «idée». La couleur parle pour elle-même, elle fait pouvoir. Il n'y a pas d' «idée» déterminante, car l'idéologie atteint le Noir dans le corps, dans son intégrité corporelle.

La visibilité des Noirs est inaltérable et permanente. Ce n'est pas une question triviale. Sur la scène publique où rien ne compte qui ne puisse se voir et s'entendre, la visibilité et le caractère audible sont de prime importance. Soutenir que ce sont purement et simplement des apparences extérieures, c'est éluder la question. Car ce sont précisément des apparences qui «apparaissent» en public, et les qualités internes, dons du cœur ou de l'esprit, ne sont politiques que dans la mesure où leur détenteur souhaite les exposer en public, les placer sous les projecteurs du marché. (Arendt, 1959: 224) 
Le Noir est Noir parce qu'il est noir. L'usage de la majuscule se montre ici particulièrement utile et indispensable. Il soutient la condition sociale et humaine qui découle de ce «caractère» surdéterminé. C'est un effet d'immanence à partir de la surface, de ce qui apparaît comme évident et immédiat. L'analyse critique de Georges Didi-Huberman de l'art minimaliste rend parfaitement compte de ce jeu de réalité: «Toujours devant cette œuvre vous voyez ce que vous voyez, toujours devant cette œuvre vous verrez ce que vous avez vu: la même chose. [...] Cela pourrait s'appeler un objet visuel tautologique» (Didi-Huberman, 1992: 33). C'est un évidement de l'image, réduite à la nature, au plus nu de ce qui est. C'est « une tentative d'éliminer toute temporalité dans ces objets de manière à les imposer comme des objets à voir toujours immédiatement, toujours exactement comme ils sont». La naturalité de ce qui apparaît objectivement sous-entend une absence de préjugé.

\section{Le Noir est pris dans l'idéologie}

Les prémisses de l'évidence, de «ce qui s’impose à l'esprit» sont bien préconstruites, préconstituées par ce que Gramsci appelle le répertoire du "sens commun». C'est un «régime de savoir», pour employer une expression foucaldienne, qui est un système d'équivalence, «le jeu d'une identité» (Foucault, 1971:37), par lequel ce qui s'affirme comme réel est réel. Or, l’immédiateté est une illusion. Le regard porté sur le Noir est une construction idéologique dont la médiation s'est dissoute par l'incorporation complexe et organisée d'un système de représentation propre à une société. Si bien que la distinction que Fanon fait entre l'apparaître et l'idée ne provient, en réalité, que d'un même processus idéologique parce que, dit Hall, la signification ne dépend plus de «ce que sont les choses", mais de la manière dont celles-ci sont signifiées. Le corps est en effet, de l'intérieur comme de l'extérieur, le lieu de rencontre de l'individu et du collectif, la matérialité de l'existence. Le «spectacle de l'autre», selon l'analyse gramscienne de Hall (2007: 81-120 et 1997: 223-279), produit un effet de réalité. Pour Colette Guillaumin, l'«apparaître» est en fait un élément secondaire de l'idéologie: «Une différence physique réelle n'existe que pour autant qu'elle est ainsi désignée, en tant que signifiant, par une culture quelconque» (2002: 96-97). La valeur de l'apparaître est sémantique, «c'est en retour qu'elle se donne pour causale». "Les catégories altérisées» sont des catégories raciales au sens latent, «dans la mesure où leur conduite, vue par la société dominante comme particulière, est considérée comme sous-tendue par un caractère somatique» (Guillaumin, 2002: 95). C'est l'alliage de la différence sociale associée à la biologisation de l'apparence physique qui forme l'efficacité de l'idéologie raciste.

\section{Une sortie politique?}

Le Noir n'existe pas. Il est invisible. C'est pourquoi, dans Les damnés de la terre (1961), l'émancipation du Noir en tant que Noir, en tant que personne, est pour Fanon impossible. Il en appelle à la lutte armée, car contre la «pure violence» du monde colonial, c'est la violence révolutionnaire qu'il lui faut opposer, réunissant une solidarité opprimée 
panafricaine et au-delà, diasporique, internationale. Il est de ce fait marxiste, mais profondément sartrien (Sartre a d'ailleurs préfacé Les damnés de la terre):

Le tiers-monde n'entend pas organiser une immense croisade de la faim contre toute l'Europe. Ce qu'il attend de ceux qui l'ont maintenu en esclavage pendant des siècles, c'est qu'ils l'aident à réhabiliter l'homme, à faire triompher l'homme partout, une fois pour toutes. (Fanon, 1961: 79)

Mais il ajoute:

Allons, camarades, le jeu européen est définitivement terminé, il faut trouver autre chose. Nous pouvons tout faire aujourd'hui à condition de ne pas singer l'Europe, à condition de ne pas être obsédés par le désir de rattraper l'Europe [...]. Tâchons d'inventer l'homme total que l'Europe a été incapable de faire triompher. (Fanon, $1961: 239-240$ )

La réification de son apparaître se fait toute entière révolte. Les damnés de la terre s'élève contre le monde colonial qu'il appelle à détruire pour le remplacer par un monde décolonisé, nouveau, fait d'une «autre espèce d'hommes» (Fanon, 1961: 29). La révolution noire, ou la révolution anticoloniale, doit être une révolution universelle, pour une démocratie postraciale. C'est une internationale pour tous les opprimés, «les forçats de la faim». Fanon vit les dernières années de sa vie en Algérie dont il a adopté la lutte et où il milite pour l'indépendance. Fanon ne veut pas un monde noir, mais un monde où chacun peut prendre place, être un homme parmi les hommes. La révolution se fait porteuse d'espoir, de création et recréation, de reconstruction à partir du rien, à partir du projet de destruction totale de la relation coloniale. Pourtant, aussi contrastant que cela puisse paraître après la lecture des Damnés de la terre, il existait néanmoins dans Peau noire, masques blancs, une idée de transformation de l'acte de création originelle:

Je ne suis pas prisonnier de l'Histoire. Je ne dois pas y chercher le sens de ma destinée. Je dois me rappeler à tout instant que le véritable saut consiste à introduire l'invention dans l'existence. (Fanon, 1952: 186)

Les damnés de la terre est un livre de guerre, précise Jean-Marie Domenach, intellectuel français et ancien résistant (Domenach, 1962). Un livre du tout ou rien dont le genre d'antagonisme poussé à l'extrême a déjà servi à des entreprises moins «louables», celles de Mussolini ou de Staline. Selon Domenach, «peut-être faut-il faire un pas de plus que Fanon, cesser de vitupérer l'Europe et son colonialisme, exister par soi-même et non plus contre d'autres».

«Par soi-même» est un bien grand mot. Domenach poursuit: «L'alliance que conclut le meilleur de l'intelligence européenne [elle fut la première à concevoir la libération de l'humanité] avec les pauvres et les opprimés, voilà ce qui continue à féconder le monde entier.» C'est pourquoi la prégnance de l'Afrique est, pour Hall, la condition de la résistance, la possibilité d'une resignification qui s'exprime par la présence de traces africaines dans le langage (vocabulaire, syntaxe, expressions...), dans les modes de vie (coutumes, traditions, mœurs sociales) et dans les diverses productions culturelles et artistiques (peintures, musique, etc.). 


\section{2) Stuart Hall et l'étranger diasporique}

\section{La barbadité ou le cinétique créole}

Stuart Hall se définit lui-même comme un métis, un hybride caribéen, aux origines africaine, écossaise, indienne et juive portugaise (Alizart et al., 2007: 77), tout d'abord considéré par Éric Macé et Éric Maigret comme un «intellectuel britannissime de la New Left» (Alizart et al., 2007: 37), l'(anti)patriarche des cultural studies, loin d'imaginer qu'ils découvriraient ensuite que celui-ci était «noir», "jamaïquain» et "immigré de l'intérieur ${ }^{24}$. Cette anecdote, que Macé et Maigret relatent dans leur livre introductif à la pensée de Hall, est intéressante, car elle résume à elle seule toute la problématique de la représentation et de l'eurocentrisme critiqués par la théorie postcoloniale. Ainsi, dit Hall, «si quelqu'un veut faire de moi l' “autre”, il peut le faire de multiples façons. [...] Je suis fait pour être Autre» (cité dans Alizart et al., 2007: 77). Un Autre emblématique de l'histoire caribéenne qui se fonde sur la «discontinuité historique»: une terre vidée et repeuplée par des populations d'origines diverses (africaines surtout, mais aussi européennes et asiatiques) à partir de l'entreprise du trafic d'esclaves. Voilà le mythe fondateur de l'identité caribéenne que Hall synthétise par cette phrase: «Notre association civile [...] commença par un acte de volonté impérial. Ce que nous appelons aujourd'hui les Caraïbes a été re-fondé dans et par une violence symbolique» (Hall, 2007: 248). Toute l'organisation des identités caribéennes se base sur cette violence originelle, où chacun est identifié par sa couleur, sa classe, son niveau d'occidentalité:

C'était donc une société soigneusement, délicatement nivelée, du plus foncé au plus clair, du blanc colonial éclatant au presque blanc, du plutôt brun au plutôt noir et au très noir, etc. Des niveaux de classe, de couleur. C'est ce qu'avaient décidé les colons. La société jamaïcaine est l'une des structures sociales les plus complexes au monde. Je savais faire ces distinctions avant même d'ouvrir les yeux [...]. Je savais quel degré sur l'échelle qui va du blanc au noir ne devait pas être dépassé. (Alizart et al., 2007: 78)

La pratique du colorisme définit l'ordre social, les limites à ne pas franchir à tous les niveaux de l'échelle des couleurs, jusqu'à un Blanc jamais atteignable malgré la meilleure impression de blancheur, car un anglicisé ne sera jamais un Anglais. Le noir qui, selon Hall, ne serait pas la marque d'une continuité anthropologique, mais celle d'une «histoire qui fut massivement supprimée, déshonorée et désavouée» (Hall, 2007: 259). C’est ce que Frantz Fanon appelait «l'expérience vécue du Noir», le noir comme signifiant du pouvoir et de la domination, mais aussi signifiant de l'absence, du vide. Un «effet de réel» de la «personnalité concrète».

\section{L'étranger diasporique}

Cette Afrique réappropriée construit l'idée d'une «nation caribéenne» comme «communauté imaginée» au-delà du territoire, et des origines réelles. Car la diaspora caribéenne interpelle la «diaspora noire» dans son ensemble. L'expérience de l'émigration

24. Hall a émigré à 18 ans de la Jamaïque vers la Grande-Bretagne. 
des Caraïbes vers les pays du «centre», ceux des colons, en particulier la GrandeBretagne, crée des relations d'affinité avec des minorités ethniques d'origines diverses. L'expérience de la diaspora, donc de la dispersion, mais aussi de la rencontre entre les dispersés, est une expérience de recomposition permanente des identités culturelles. La barbadité, l'africanité ou encore l'indo-occidentalité peuvent rassembler des individus qui ne correspondent pas physiquement, dans leur apparaître social, à la description suggérée par les termes culturels. Hall cite un artiste qui se décrit lui-même comme «un artiste masculin anglo-américain, créole, indo-occidental, trinidais et indien de la postindépendance, élevé dans le christianisme» et qui relate une anecdote où des «gens instruits» ne comprenaient pas qu'il soit originaire des Caraïbes alors qu'il «a l'air Asiatique» (Hall, 2007: 261). L’origine est un réel «tourbillon», réappropriée, transformée, recomposée à partir d'expériences et de récits divers: «Les cultures, bien entendu, ont leurs “localisations". Mais il n'est plus guère facile de dire d'où elles viennent » (Hall, 2007: 255).

Ainsi, bien que les migrations de retour vers les Caraïbes soient un phénomène notable, l'expérience de la diaspora est bien plus qu'une relation transnationale entre les Caribéens et les émigrés installés en Grande-Bretagne, entre un lieu d'origine et un lieu d'installation. L'individu diasporique est transnational, extraterritorial. C'est un étranger par excellence, mais par lequel le dedans et le dehors sont transfigurés. La diaspora est une re-création, une reformulation des origines et des identités culturelles. L'expérience diasporique met l'accent sur la nature profondément dynamique et dialogique de la culture où les rapports de pouvoir et d'autorité sont certes prégnants, mais où aussi, le dominé peut trans-former le dominant, et où globalement l'expression de «l'authenticité» n'a plus de sens. La créolisation est ce mouvement de passage et de désarticulation des signifiants de la domination. Ce pourquoi la langue, le langage sont tellement importants dans toutes les études sur l'idéologie. Hall engage et encourage une politique de l'existence, mais ce n'est pas dans le sujet qu'il voit la possibilité de la nouveauté et de la pluralité, mais dans les productions culturelles, dans l'art, dans «des forces diffuses » qui «échappent au système mondial actuel» (Alizart et al., 2007: 91).

La posture postcoloniale rompt avec une tradition occidentale de mise en silence de soi pour représenter l'universel. Elle réitère la perspective, initiée déjà par la pensée des exilés, des intellectuels juifs d'Europe occidentale (Arendt, mais aussi Walter Benjamin, Theodor Adorno, Siegfried Kracauer, etc. $)^{25}$, à une pensée politique et critique qui prend la parole «au nom de», au sein même du savoir légitime et «neutre». La déconstruction de l'illusion de l'essence et du naturel des cultures et des nations ne conduit pas à une indétermination totale des individus et de l'humanité. Elle témoigne au contraire d'une volonté de conscience et d'action à partir de sa propre place, spécifique, différente. À partir d'un récit tragique et d'une réflexion poussée sur les pratiques de la domination et le poids de l'hégémonie, Hall parvient à mettre en lumière 
les espaces de batailles et de lutte par lesquels les opprimés se réapproprient une liberté. La position diasporique reste une position en marge, mais fondamentalement moderne, qui expérimente le vécu de la globalisation dans leur corps et dans leur existence. Ainsi, l'identité noire revendiquée devient une identité politique. Une identité qui ne se borne pas (nécessairement) dans un mythe de pureté raciale, mais qui, au contraire, porte la pluralité essentielle à toute humanité. Ce n'est plus de la survivance, mais de la création. À l'instar d'Hannah Arendt qui, à la question: «Qui êtes-vous?», répondait: «Une Juive», à la question: «Es-tu noir?», Hall répond: «Oui, bien sûr, je suis noir! Je suis un intellectuel noir!» (Alizard et al., 2007: 78).

\section{CONCLUSION}

La nécessité de la naissance situe l'individu dans le monde, dans ses espaces d'action, dans ses rapports de pouvoir, dans ses modalités de lien politiques. Dire qu'il y a une naissance au monde nécessaire, ce n'est pas réduire les identités à une essence fixe, déterminée. Rien ne précède la naissance, dans le sens où le renouvellement n'est conditionné par aucune loi, aucun ordre. Elle ne présage de rien. Une naissance qui, socialement, a des qualités visibles, identifiables, nommables, mais dont le devenir est ouvert. Le minoritaire porte un nom qui fait sens, qui agit et porte à conséquences. C'est parce qu'il n'y a rien d'anodin à cette situation qu'elle est précisément de l'ordre de l'existence. Une existence qui n'est pas politique en soi, mais d'où peut émerger une conscience «d'être au monde» particulière.

Cette conscience, quelle qu'elle soit, concerne certes tout individu, mais cela ne signifie pas qu'il y ait équivalence. Parce qu'il y a une violence, une oppression, une relation de pouvoir intrinsèque à l'émergence de la question minoritaire, quand cette question arrive à se poser, l'ordre idéologique est déjà bousculé. Le politique du minoritaire est ce qui est dans l'espace d'entre-deux. Ce qui crée sa spécificité, c'est sa place, celle d'être dans deux endroits à la fois, normatif et particulier. C'est pourquoi Arendt peut dire à Jaspers qu'ils ne sont pas sur le même bateau. C'est pourquoi aussi, Benny Lévy, au contraire de Sartre, peut s'engager dans un autre ailleurs éthico-politique. Fanon ne parvient pas, ou refuse, d'entrer dans l'entre-deux. Sa rupture est totale et propose une politique du néant pour recréer l'espace-qui-est-entre-les-hommes. L'identification d'un nom présume que l'on s'attend à quelque chose. Or, il n'y a rien de plus déstabilisant qu'un nom qui dit qu'il n'est pas ce qu'il est. Le discours de Monique Wittig sur La Pensée straight, prononcé en 1978, a profondément révolutionné le point de vue féministe, comme tout point de vue réceptif à cette politique, par sa fameuse conclusion: «Les lesbiennes ne sont pas des femmes» (Wittig, 1992: 61). Wittig s'attaque, à l'espace global, à la mise en évidence d'un «régime politique». Car l'apparition du nom oblige le sans-nom à se nommer, à se reconnaître. En désidentifiant la lesbienne, Wittig crée l'espace vide dans le sens commun, non pas celle reléguée par la norme, subie, mais celle conquise par une sémiologie politique. Hall, pour sa part, incarnerait presque la déconstruction essentialiste par une sur-représentation de la 
pluralité. Le minoritaire devient politique quand il crée une rupture avec le majoritaire, ou avec le pouvoir, le «régime», l'évidence idéologique.

Dans l'espace commun, l'apparition minoritaire est une perpétuelle remise en question du mode d'être normatif, c'est-à-dire, «qui va de soi». Par la lutte armée, la lutte symbolique, le langage, le dialogue et les engagements de toute autre nature, cette apparition va jusqu'à en transformer les repères. L'ordre apparent du majoritaire est détourné par la variance des réappropriations du nom minoritaire. Le nom se fait politique et réintroduit la pluralité dans le fantasme du monde global commun.

Le monde globalisé, le monde des sans-noms, semble s'orienter à la fois vers une uniformisation accrue des sociétés et des réseaux économiques formés autour des modèles dominants (européens, nord-américains), et vers une explosion identitaire des subjectivités. Il s'inscrit aussi dans une perte du sens du monde entre les hommes, dont l'un des projets communs actuels les plus porteurs est tourné vers la protection de l'humanité, par celle de la nature, le projet de l'écologie, appuyé par des scénarii de catastrophes, voire de fin du monde. Le projet commun est dépolitisé. Il relève de la survie.

La récente élection de Barack Obama à la présidence des États-Unis constitue en cela une révolution. Il ne dit plus «Black is beautiful», mais ouvre la possibilité de dire: "Je ne vous ressemble pas [sous-entendu, vous = le neutre indéterminé], mais je peux vous représenter.» Sa révolution lui a valu un prix Nobel de la paix alors même qu'il venait de commencer son mandat. Pour être élu, il a dû déployer une force communicationnelle extraordinaire de ses «dons du cour et de l'esprit». Il est le Juif d'exception de Hannah Arendt, un «spécimen d'humanité», beau, cosmopolite, intelligent, diplômé de Harvard, la plus prestigieuse université américaine et internationale. Pour combler cette distance avec le pouvoir, il porte la responsabilité sacrificielle de l'humanité entière sur sa personne.

Yes we can! Pour être Noir et président, il faut d'abord être un super héros. Son génie, néanmoins, a été de réussir à toucher et à rassembler le monde entier. Son nom a donné une visibilité dans l'ordre anonyme des subjectivités libres et indéfinies. À la grande indétermination de la masse globale, les noms apparaissent avec un projet éthique et politique.

L'espoir se trouve dans la rencontre, dans le passage, dans l'itinéraire de passeurs de frontières.

\section{RÉSUMÉ}

Cet article propose de mener une réflexion sur la tension dialectique minoritaire/majoritaire dans une analyse théorique, littéraire et microsociale de l'«apparaître » du minoritaire l'apparaître étant le «qui» dans le «qui suis-je» qui se déclare ou se signifie dans l'espace social et public. II s'agira d'appréhender les figures du Juif et du Noir comme des idéaltypes du minoritaire afin d'élaborer une dimension politique spécifique du minoritaire, qui se compose au sein d'une citoyenneté républicaine, individuelle, égalitaire et anonyme. Tout d'abord, nous nous efforcerons d'inscrire cette étude dans une perspective de sociologie politique et morale. Puis, afin de percevoir et d'analyser comment se discute et s'élabore le fait politique et ce qu'implique 
l'apparition du minoritaire dans le champ social, nous étudierons des textes choisis parmi certains dialogues, correspondances et essais dans le champ des études postcoloniales (Fanon, Hall) et de la «question juive» (Arendt, Sartre).

\section{ABSTRACT}

This article proposes to reflect upon the dialectical tension between minority and majority to the level of theoretical, literary, and microsocial analysis of the "appearance" of the minorityappearance being the "who" in the "who am I?" that declares and signifies itself in social and public space. It aims to grasp the figures of the Jew and the Black as ideal types of the minority in order to elaborate upon a specific political dimension of the minority that is constituted within the individuality, equality and anonymity of a republican citizenship. First of all, we will endeavor to carry out this study from the perspective of political and moral sociology. In order to perceive and analyze how political activity is discussed and elaborated and to see what is implicated in the appearance of the minority in the social field, we will study selected texts among certain dialogues, correspondences and essays in the field of post-colonial studies (Fanon, Hall) and the "Jewish question" (Arendt, Sartre).

\section{RESUMEN}

Este artículo propone desarrollar una reflexión acerca de la tensión dialéctica minoritariomayoritario, en un análisis teórico, literario y microsocial del "mostrarse" del individuo del minoritario, donde mostrarse es el "quien" en el "quién soy yo", quien se declara o se anuncia en el espacio social y público. Se trata de aprehender las figuras del Judío y del Negro como idealtipos del minoritario con el fin de elaborar una dimensión política específica del mismo al interior de una ciudadanía republicana, individual, igualitaria y anónima. Inicialmente nos esforzaremos por enmarcar este estudio en una perspectiva de la sociología política y moral. A continvación el fin de percibir y analizar cómo se discute y se elabora el hecho político y qué implica la aparición del minoritario en el campo social, estudiaremos algunos textos escogidos, entre ellos ciertos diálogos, correspondencias y ensayos en el campo de los estudios postcoloniales (Fanon, Hall) y de la "cuestión judía" (Arendt, Sartre).

\section{BIBLIOGRAPHIE}

Alizart, M., S. Hall, É. Macé et É. Maigret (2007), Stuart Hall, Paris, Amsterdam.

Arendt, H. (2005), Responsabilité et jugement, Paris, Payot.

Arendt, H. (2001 [1993]), Qu'est-ce que la politique?, Paris, Seuil.

Arendt, H. et K. JAspers (2006 [1985]) «La philosophie n'est pas tout à fait innocente», Paris, Payot \& Rivages. Rivages.

Arendt, H. (2005 [1959]), «Réflexions sur Little Rock», in Responsabilité et jugement, Paris, Payot.

Arendt, H. (1994 [1958]), Rahel Varnhagen. La vie d'une juive allemande à l'époque du romantisme, Paris, Calmann-Lévy.

Arendt, H. (1994 [1958]), La condition de l'homme moderne, Paris, Calmann Lévy.

Arendt, H. (2005 [1954]), La crise de la culture. Huit exercices de pensée politique, Paris, Gallimard.

Arendt, H. (1984 [1951]), Les Origines du totalitarisme, I, Sur l'antisémitisme, Paris, Seuil.

Arendt, H. (2002 [1948]), Les Origines du totalitarisme, suivi de (1963) Eichmann à Jérusalem, Paris, Gallimard. Arendt, H. (1993 [1946]), La tradition cachée. Le Juif comme paria, Paris, Christian Bourgeois.

Bernstein, R. J. (1996), Hannah Arendt and the Jewish Question, Cambridge, Massachusetts, The MIT Press. Bнавна, H. (2001 [1994]), The Location of Culture, New York/Londres, Routledge. 
Brodkin, K. (1999), How Jews Became White Folks and What That Says About Race in America, Rutgers, Rutgers University Press.

Didi-Huberman, G. (1992), Ce que nous voyons, ce qui nous regarde, Paris, Les Éditions de Minuit.

Domenach, J-M. (1962), «Les damnés de la terre (II)», in «Les Antilles avant qu'il soit trop tard», Esprit, avril, no 4, p. 634-645. <http://www.esprit.presse.fr/review/article.php?code=1982>, consulté le 20 décembre 2009.

ELias N. et J. L. SCOTson (2001 [1965]), Logiques d'exclusion: enquête sociologique au cour des problèmes d'une communauté, Paris, Calmann-Lévy.

Facendis, D. de (2003), «Hannah Arendt et le mal», in Dagenais, D. (dir.). Hannah Arendt, le totalitarisme et le monde contemporain, Saint Nicolas, Presses de l'Université Laval, p. 52-102.

Fanon, F. (2003 [1961]), Les damnés de la terre, Paris, Gallimard, La Découverte.

Fanon, F. (1971 [1952]), Peau noire, masques blancs, Paris, Seuil.

Foucault, M. (2004 [1976]), Histoire de la sexualité, I, La volonté de savoir, Paris, Gallimard.

Foucault, M. (2003 [1971]), L'ordre du discours, Paris, Gallimard.

Guillaumin, C. (2002), L'idéologie raciste. Genèse et langage actuel, Paris, Gallimard.

Hall, S. (2007), Identités et cultures. Politiques des cultural studies, Paris, Amsterdam.

HaLL, S. (1997), "The spectacle of the "other" ", in HaLL, S. (dir.). Representation: Cultural Representations and Signifying Practices, Sage/Open UP, p. 223-279.

Ignatiev, N. (1996), How the Irish Became White, Londres, Routledge.

Lapierre, N. (2009), «Lévi-Strauss: le regard rapproché d'Isac Chiva», Mediapart. Mis en ligne le 5 novembre 2009. (Réactions de Pierre Ferron, respectivement le 6 novembre et le 8 novembre 2009), $<$ http://www.mediapart.fr/club/blog/nicole-lapierre/051109/levi-strauss-le-regard-rapproche-d-isacchiva>, consulté le 20 décembre 2009.

Leiвovici, M. (1998), Hannah Arendt, une Juive — Expérience, politique et histoire, Paris, Desclée de Brouwer. Levinas, E. (2006 [1963]), Difficile liberté. Essai sur le judaïsme, Paris, Albin Michel.

LÉvy, B. (2003), Être juif. Étude lévinasienne, Paris, Verdier.

LÉvy, B. (2002), Le meurtre du Pasteur. Critique de la vision politique du monde, Paris, Verdier-Grasset.

Maurin É. (2004), Le ghetto français. Enquête sur le séparatisme social, Paris, Seuil.

Morgensztern I. (2008), Benny Lévy. La révolution impossible (La loi du retour), France-Allemagne, coproduction SZ Productions/Arte/Ina.

Ouaknin, M.-A. (2003 [1992]), Méditations érotiques. Essai sur Emmanuel Levinas, Paris, Payot \& Rivages.

Poliakov L. (1968), Histoire de l'antisémitisme, tome III, De Voltaire à Wagner, Paris, Calmann-Lévy.

SArtre, J.-P. et B. LÉVY (1991), L'espoir maintenant. Les entretiens de 1980, Paris, Verdier.

SARTRE, J.-P. (2005 [1946]), Réflexions sur la question juive, Paris, Gallimard.

SChofield, C. (2003), «Itinéraire d’un écrivain engagé. Richard Wright le subversif», Le Monde diplomatique, août, no 593, p. 25, <http://www.monde-diplomatique.fr/2003/08/CORYELL/10358>, consulté le 20 décembre 2009.

Simmel, G. (1984 [1908]), «Digression sur l'étranger», in Grafmeyer, Y. et I. Joseph (dir.). École de Chicago. Naissance de l'écologie urbaine, Paris, Aubier, p. 53-59.

Simon, P.-J. (2006), Pour une sociologie des relations interethniques et des minorités, Rennes, Presses Universitaires de Rennes.

Tocqueville, A. de (1981 [1840]), De la démocratie en Amérique II, Paris, Garnier-Flammarion.

Tocqueville, A. de (2000 [1835]), De la démocratie en Amérique I, Paris, Gallimard.

Traverso, E. (2004), La pensée dispersée. Figures de l'exil judéo-allemand, Paris, Léo Scheer.

Traverso, E. (1997), Les marxistes et la question juive, Paris, Kimé.

Wittig, M. (2007 [1992]), La Pensée straight, Paris, Amsterdam.

Wright R. (1974 [1945]), Black Boy, Paris, Gallimard.

Yerushalmi, Y. H. (1991 [1982]), Zakhor — Histoire juive et mémoire juive, Paris, Gallimard.

Young-Bruehl, E. (1999 [1982]), Hannah Arendt. Biographie, Paris, Calmann-Lévy. 